\title{
A new variation of the Buckingham exponential-6 potential with a tunable, singularity-free short-range repulsion and an adjustable long- range attraction
}

\author{
Jasper C. Werhahn ${ }^{a}$, Evangelos Miliordos ${ }^{b}$ and Sotiris S. Xantheas ${ }^{b, *}$ \\ ${ }^{a}$ Physik-Department E11, Technische Universität München, James-Franck-Strasse, D- \\ 85748 Garching, Germany \\ ${ }^{b}$ Physical Sciences Division, Pacific Northwest National Laboratory, 902 Battelle \\ Boulevard, P.O. Box 999, MS K1-83, Richland, WA 99352
}

\begin{abstract}
We introduce new generalized (reverting to) and extended (not reverting to the original) 4-parameter forms of the (B-2) Potential Energy Functional (PEF) of Wang et al. [ $J$. Chem. Theor. Comp. 9, 452 (2013)], which itself is a modification of the Buckingham exponential-6. The new forms have a tunable, singularity-free short-range repulsion and an adjustable long-range attraction and are capable of describing even the strong iondipole interactions present in the halide- and alkali metal-water dimers. They are slightly better than the 4-parameter generalized Buckingham exponential-6 $(\mathrm{gBe}-6)$ and of comparable quality with the 4-parameter extended Morse (eM) PEFs introduced recently by us.
\end{abstract}


* Corresponding author: E-mail: sotiris.xantheas@pnnl.gov

The modified exponential-6 Buckingham Potential Energy Function (PEF), $V_{B e-6}$ or $(B e-6)$, is a three-parameter function of the form

$$
V_{B E-6}(r ; A, B, C)=A e^{-E r}-\frac{c}{r^{\bar{\varepsilon}}},
$$

i.e. a repulsive exponential and an attractive $1 / r^{6}$ term of the internuclear distance $r$. It was introduced by R. A. Buckingham ${ }^{[1]}$ in 1938 as an alternative to the Lennard-Jones $(L J)$ potential, ${ }^{[2-4]}$ replacing the $1 / r^{12}$ term of that PEF with the Born-Mayer expression ${ }^{[5,6]}$ to study the equation of state for gaseous $\mathrm{He}, \mathrm{Ne}$ and Ar. The designation "modified" indicates that the additional $1 / r^{8}$ term present in the original potential, albeit with the same number of parameters, has been dropped. This PEF has been extensively used in recent years ${ }^{[7-12]}$ to describe weak(er) intermolecular interactions such as the ones in $n$ alkanes and/or between non-polar molecules in order to simulate phase equilibria. In its present form it is not suitable for modeling stronger interactions, such as ion-dipole, due to its fixed $1 / r$ dependence.

The (Be-6) PEF can be cast in the form

$$
V_{B e-6}\left(r ; \varepsilon, r_{m}, \alpha\right)=\frac{\varepsilon}{1-\frac{6}{\alpha_{B}}}\left[\frac{6}{\alpha_{B}} e^{\alpha_{B}\left(1-\frac{r}{r_{m}}\right)}-\left(\frac{r_{m}}{r}\right)^{6}\right]
$$

in terms of the minimum distance $r_{m}$ and the well depth $\varepsilon$. The parameters $(A, B, C)$ of equation (1) are related to $\left(\varepsilon, r_{m}, \alpha\right)$ via

$$
A=\frac{6 \varepsilon \cdot e^{\alpha}}{\alpha-6}, \quad B=\frac{\alpha}{r_{m}}, \quad C=\frac{\varepsilon \cdot \alpha}{\alpha-6} r_{m}^{6}
$$


The potential "turns over" for small $r$ and it is necessary to add a "hard wall" for distances smaller than a "cutoff" distance $\left(R_{\text {cut }}\right)$, being the smallest positive value of $r$ for which $d V_{B z-6}(r) / d r=0$, viz.

$$
\left(\frac{R_{\text {cut }}}{r_{\mathrm{m}}}\right)^{7} e^{\alpha_{D}\left(1-\frac{B_{\text {sut }}}{r_{\mathrm{m}}}\right)}=1
$$

Note that equation (2) has also been used with a coefficient $c$ in front of the $\left(\frac{r_{m}}{r}\right)^{6}$ term. In that case there are 4 parameters but $\varepsilon$ corresponds to the well depth only for $c=1$, viz. eq. (2).

The (Be-6) PEF, equation (2), can be cast in terms of the reduced coordinates $\varepsilon^{*}=\frac{V_{E g-\epsilon}}{z}$ and $r^{*}=\frac{r}{r_{m 2}}$ in its scalable form ${ }^{[13]}$

$$
\varepsilon_{b e-6}^{*}\left(r^{*} ; \alpha\right)=\frac{1}{1-\alpha}\left[\alpha e^{\frac{6\left(1-r^{*}\right)}{\alpha}}-\left(\frac{1}{r^{*}}\right)^{6}\right]
$$

where $\alpha=\frac{6}{\alpha_{B}}$ and singularities exist at $r^{*}=0$ and for $\alpha=1$.

In a recent publication ${ }^{[13]}$ we described a procedure of extending scalable PEFs by adding one additional parameter producing either their generalized (reverting to the original for some value of the additional parameter) or extended (not reverting to the original for any value of the additional parameter) forms and applied it to the Mie, Lennard-Jones, Morse and (Be-6) PEFs. Among the PEFs introduced in our earlier study, ${ }^{[13]}$ the 4-parameter generalized Buckingham exponential- 6 or $(g B e-6)$ PEF is

$$
\varepsilon_{g B e-6}^{*}\left(r^{*} ; \alpha, \delta\right)=\frac{\alpha \delta}{6(1-\alpha)}\left[e^{\frac{6\left(1-r^{*}\right)}{\alpha}}-\left(\frac{1}{r^{*}}\right)^{6}\right]-\left(\frac{1}{r^{*}}\right)^{\delta},
$$

reverting back to the original 3-parameter $(B e-6)$ for $\delta=6$. A hard wall still needs to be added for $r^{*}<R_{\text {cut }}^{*}$, where $R_{\text {cut }}^{*}$ is numerically found (for a fixed value of $\alpha$ ) as the lowest positive solution (different than $r^{*}=1$ ) of the equation

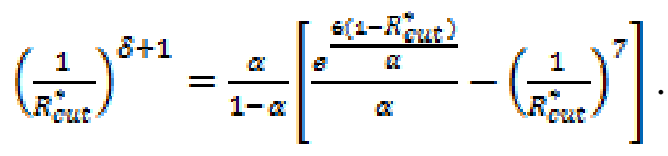


Wang et al. ${ }^{[14]}$ have recently corrected the problem with the "turning-over" of the repulsive wall of the (Be-6) PEF by introducing the "Buffer-2" potential, $(B-2)$, which has a tunable repulsion with no singularity at $r=0$. Compared to the original $(B-2)$ formula ${ }^{[14]}$ the parameter $\sigma=r_{m}$ and the PEF can be written in a scalable form as

$$
V_{B-2}\left(r^{*} ; \gamma\right)=\frac{2}{1-\frac{3}{\gamma+3}}\left(\frac{1}{1+\left(r^{*}\right)^{6}}\right)\left[\frac{3}{\gamma+3} e^{\gamma\left(1-r^{*}\right)}-1\right] .
$$

Note that $\gamma>0$, otherwise the (B-2) PEF diverges for large values of $r$.

In this paper, inspired by the work of Wang et al., ${ }^{[14]}$ and following our previous procedure ${ }^{[13]}$ we introduce the generalized and extended forms of the $(B-2) \mathrm{PEF}$.

The generalized 4-parameter form $(g B-2) \mathrm{PEF}$ is

$$
\varepsilon_{g B-2}^{*}\left(r^{*} ; \gamma, \delta\right)=\frac{\delta+2 \gamma+6}{2 \gamma} \cdot \frac{1}{1+\left(r^{*}\right)^{6}}\left[\frac{6+\delta}{\delta+2 \gamma+6} e^{\gamma\left(1-r^{*}\right)}-1\right]-\frac{1}{1+\left(r^{*}\right)^{\delta}},
$$

reverting to the original $(B-2) \mathrm{PEF}$ for $\delta=6$. It has a tunable short-range repulsion with no singularity at $r=0$ and in addition an adjustable long-range attraction.

Similarly, the extended 4-parameter form $(e B-2)$ is

$$
\varepsilon_{e B-2}^{*}\left(r^{*} ; \gamma, \zeta\right)=\frac{2 \zeta}{\gamma} \cdot \frac{1}{1+\left(r^{*}\right)^{6}}\left[e^{\gamma\left(1-r^{*}\right)}-1\right]-\frac{1}{\left(r^{*}\right)^{\zeta}}
$$

which also has a tunable short-range repulsion and an adjustable long-range attraction, albeit with a singularity at $r=0$. We remind readers that the coefficients of the $(g B-2)$ and $(e B-2)$ PEFs are determined by the requirement to satisfy the scalability constraints, ${ }^{[13]}$ namely $\left.\varepsilon^{*}\left(r^{*}\right)\right|_{r^{*}=1}=-1, \lim _{r^{*} \rightarrow \infty} \varepsilon^{*}\left(r^{*}\right)=0$ and $\left.\frac{\partial z^{*}}{\partial r^{*}}\right|_{r^{*}=1}=0$. The $(e B-2)$, as it is the case for the $(g B e-6)$, turns over at small $r^{*}$ for some choices of the parameters $\gamma$ and $\zeta$, so a hard wall has to be inserted at the lowest solution of the equation

$$
\frac{\gamma}{2} \frac{1+R_{\text {cut }}^{*}}{R_{\text {cut }}^{*} t+1}=e^{\gamma\left(1-R_{\text {cut }}^{*}\right)}\left(6 R_{\text {cut }}^{*}+\gamma\right)-6 R_{\text {cut }}^{*}
$$

As mentioned earlier, the (Be-6) PEF has been traditionally used to model weak intermolecular interactions, which at long range vary as $1 / r^{6}$, and it is therefore 
inappropriate for describing other types of interactions with a different $1 / r$ dependence, such as for instance ion-dipole interactions. We have previously ${ }^{[15]}$ used the scalable form of the ( $g B e-6)$ PEF, equation (4), to fit the halide- and alkali metal-water interactions obtained from high level $a b$ initio calculations. We found that the ( $g B e-6)$ PEF produced very accurate fits to the ab initio data that were between one and two orders of magnitude better in the $\chi^{2}$ than the original (Be-6) PEF. This was because the $(g B e-6)$, due to its enhanced flexibility arising from the additional parameter that allows for a variable $1 / r$ dependence, was found to describe not only the long-range but also the minimum and repulsive wall parts of the respective potential energy surfaces (PESs) quite accurately.

In this study we test the performance of the 3-parameter $(B-2)$ and the newly introduced 4-parameter $(g B-2)$ and $(e B-2)$ forms by fitting the same set of ab initio data for the halide- and alkali metal-water interactions and compare the results with the ones obtained previously ${ }^{[15]}$ with the $(B e-\emptyset),(g B e-\emptyset)$ and extended Morse $(e M)$ PEFs - the later is included in the comparison since it was found to be superior among all PEFs for fitting the ab initio data in those earlier studies. The form of the (eM) PEF introduced earlier $^{[13]}$ is

$$
\varepsilon_{e M}^{*}\left(r^{*} ; \alpha_{M}^{*}, \gamma_{2}\right)=\frac{\gamma_{2}}{\alpha_{M}^{*}}\left[e^{2 \alpha_{M}^{*}\left(1-r^{*}\right)}-e^{\alpha_{M}^{*}\left(1-r^{*}\right)}\right]-\left(\frac{1}{r^{*}}\right)^{\gamma_{2}},
$$

where $\alpha_{M}^{*}=\alpha_{M} r_{m}$ and $\alpha_{M}, \gamma_{2}$ are constants.

Details of the level of electronic structure theory and basis sets used to obtain the $a b$ initio data as well as the fitting process are described previously. ${ }^{[15]}$ The fits are performed for the Relaxed Approach Paths (RAPs) obtained by fixing the X/M - O distance and optimizing the rest of the internal coordinates. The scaled $\left(\varepsilon^{*}, r^{*}\right) a b$ initio data for each system were weighted by the function

$$
w\left(r^{*}\right)=\frac{1}{e^{-\zeta\left(r^{*}-\eta\right)}+1}
$$

to balance the fit between the repulsive and long-range regions. The parameter $\xi$ determines the steepness of $w\left(r^{*}\right)$ in the [0,1] range, whereas $\eta$ controls the $r^{*}$ value for 
which $w(\eta)=0.5$. The smaller the value of $\xi$, the larger part of the repulsive wall is taken into account in the fit. In the current fits we used the values of $\eta=0.85$ and $\xi=75$.

The quality of the fits was measured by

$$
\chi^{2}=\frac{\Sigma_{i} w^{2}\left(r_{i}^{*}\right)\left[s_{i}^{*}-f\left(r_{i}^{*}\right)\right]^{2}}{\sum_{i} w^{2}\left(r_{i}^{*}\right)}
$$

where $\varepsilon_{i}^{*}$ are the scaled $a b$ initio binding energies and $f$ stands for the PEF being evaluated. Needless to emphasize that $\chi^{2}$ will largely depend on the chosen values of $\eta$ and $\xi$ used in the fit, see the discussion in our earlier publication. ${ }^{[15]}$

The behavior of the various PEFs is shown in Figure 1 for values of the parameters that are close to the fitted values for the halide- and alkali metal-water dimers (cf. Tables II and IV of Reference ${ }^{[15]}$ ). The [left] / [right] panels of Figure 1 show the behavior of the $[(B e-6),(g B e-6)] /[(B-2),(g B-2)$ and $(e B-2)]$ PEFs compared to the $(e M)$ PEF. In the top panels of Figure 1 the parameters were chosen in an attempt to have the same repulsive wall (to the degree possible) for all potentials and show the variation in the area around the minimum and long-range parts. Contrary, in the bottom panels of Figure 1, an attempt was made to have a similar minimum plus long-range behavior (as much as possible) for all PEFs and show the difference in the short-range part of the potential for that choice of the potential parameters. The examples shown in Figure 1 clearly indicate the major differences among the various PEFs considered in this study: PEFs that have almost the same repulsion differ widely in the long range-part and vice versa. At the same time they demonstrate the delicate balance between the simultaneous fitting of the repulsive and the long-range parts and unambiguously exemplify the dilemma in choosing to assign more importance in either the short-range repulsive or long-range attractive part of the potential.

Table 1 shows the results of the fits (using $\eta=0.85$ and $\xi=75)$ for the $\mathrm{X}^{-}\left(\mathrm{H}_{2} \mathrm{O}\right)$, $\mathrm{X}=\mathrm{F}, \mathrm{Cl}, \mathrm{Br}$, I, while Table 2 the corresponding ones for the $\mathrm{M}^{+}\left(\mathrm{H}_{2} \mathrm{O}\right), \mathrm{M}=\mathrm{Li}, \mathrm{Na}, \mathrm{K}, \mathrm{Rb}$, $\mathrm{Cs}$, series. We note that the results with the $(e M),(B e-\sigma)$ and $(g B e-\sigma)$ PEFs are slightly different than the ones from our previous publication. ${ }^{[15]}$ The $(B-2)$ PEF performs poorly in fitting the $a b$ initio data for both systems as indicated by the value of $\chi^{2}$ and the fact 
that the fitted value of $\gamma$ (for $\eta=0.85$ and $\xi=75$ ) is negative, which makes it turn over for large values of $r$. The ( $g B-2)$ PEF improves the fits by 1 to 2 orders of magnitude whereas $(e B-2)$ is even slightly better for some systems. When compared to the fits with the (eM) PEF, the $(e B-2)$ is slightly better for the $\mathrm{X}^{-}\left(\mathrm{H}_{2} \mathrm{O}\right)$ and slightly worse for the $\mathrm{M}^{+}\left(\mathrm{H}_{2} \mathrm{O}\right)$ series. Also shown in Tables 1 and 2 are the results of the fits when forcing the longrange behavior to be exactly $1 / r^{2}$, i.e. setting $\delta=2.0$ for $(g B-2)$ and $\zeta=2$ for the $(e B-2)^{*}$ PEFs. This has only a minor effect on the values of both the other parameter $(\gamma)$ and $\chi^{2}$, an observation that is consistent with the fact that the fitted values of both $\delta$ and $\zeta$ are quite close to 2 when these parameters are allowed to vary. Using a variable long range exponent results in a slightly better fit and can be viewed as the price one has to pay for simultaneously fitting both the long-range, minimum and short-range parts of the potential with such a limited number of parameters.

The quality of the fits listed in Tables 1 and 2 are graphically illustrated in Figures 2 [for $\mathrm{X}^{-}\left(\mathrm{H}_{2} \mathrm{O}\right)$ ] and 3 [for $\left.\mathrm{M}^{+}\left(\mathrm{H}_{2} \mathrm{O}\right)\right]$. The value of $\gamma=0.004$ is used for the fits of the $(B-$ 2) PEF in those 2 figures, since we could not find a value of $\gamma>0$ to best fit the data. We note the inability of the ( $B-2)$ to fit (for $\eta=0.85$ and $\xi=75$ ) both the short-range repulsive and long-range attractive parts of the PESs simultaneously; in contrast, both $(g B-2)$ and $(e B-2)$ PEFs perform extremely well in those two regions.

As was noted earlier, the choice of values for $\eta$ and $\xi$ have quite a large effect on the overall fit of the PESs, as they determine the weights for each data point via eq. (10). In Figure 4 we illustrate this effect for the $\mathrm{Br}^{-}\left(\mathrm{H}_{2} \mathrm{O}\right)$ PES. In the left panel we show the fits (and corresponding values of $\chi^{2}$ ) for $\xi=0$ (for which the value of $\eta$ is irrelevant), i.e. putting emphasis in fitting the repulsive wall. Indeed for that case all 3 PEFs have quite similar repulsive walls, however the $(B-2)$ fails in describing the long-range part unlike the $(g B-2)$ and (eB-2) PEFs. The middle panel shows the fits for $\xi=75$ and $\eta=0.85$ used to derive the fits in Table 1 and Figure 2. For that choice of the parameters of the weighting function, both the $(g B-2)$ and the $(e B-2)$ PEFs perform better in the long-range part and the $\chi^{2}$ is smaller than in the left panel (although the absolute value is altered by the fact that points high up in the repulsive wall are included with zero weights). Finally, in the 
right panel of Figure 2 the fits using $\xi=1000$ and $\eta=0.5$, putting emphasis in fitting the long-range attractive part, are shown. Indeed in that case the long-range part is slightly better than that in the middle panel (and the repulsive wall slightly worse), while the value of $\chi^{2}$ is even smaller than before since the repulsive wall is not included in its calculation. These illustrative examples clearly demonstrate the caution that should be taken in using those PEFs to fit PESs and the need to have a balanced description of both the short- and long-range parts.

In conclusion, we have introduced the generalized $(g B-2)$ and extended $(e B-2) 4-$ parameter forms of the (B-2) PEF of Wang et al. ${ }^{[14]}$, which itself is a modification of the Buckingham exponential- 6 potential. The new forms have a tunable, singularity-free (save the (eB-2) PEF) short-range repulsion and an adjustable long-range attraction. The later is essential in allowing them to fit the 1-dimensional RAPs of the strong (ion-dipole) halide- and alkali metal-water interactions. Overall, the new forms of the potentials produce fits that are between 1 and 2 orders of magnitude better than the 3-parameter ( $B$ 2) PEF, slightly better than the 4-parameter generalized Buckingham exponential-6 and of comparable quality with the 4-parameter extended Morse $(e M)$ potential energy functions for these systems. The role of the parameters $\gamma$ and $\delta$ of the $(g B-2)^{*}$ PEF, equation (6), is graphically illustrated in Figure 5: the parameter $\gamma$ mainly controls the repulsive wall, whereas $\delta$ the long-range part of the PES. By allowing $\delta$ to vary, a slightly better fit is obtained with respect to the case when $\delta=2$ for the present case and this is because an overall better fit of the short-, long-range and minimum areas of the potential can thus be obtained. It is anticipated that the flexibility of the newly introduced forms in both the short- and long-range parts of the PES, in conjunction with their singularity-free behavior at short distances, will enable them to accurately model a wide spectrum of intermolecular interactions ranging from the very weak to the very strong including those in between. This proposition will be the subject of future studies.

Acknowledgment. JCW thanks the International Max Planck Research School on Advanced Photon Science for a graduate fellowship and the US Department of Energy for additional financial support during his visit to PNNL. SSX acknowledges the support, 
via a Visiting Fellowship, of the Technische Universität München at Garching - Institute for Advanced Study, funded by the German Excellence Initiative and Prof. A. Laubereau for his hospitality. This work was supported by the US Department of Energy, Office of Science, Office of Basic Energy Sciences, Division of Chemical Sciences, Geosciences \& Biosciences. Pacific Northwest National Laboratory (PNNL) is a multi-program national laboratory operated for DOE by Battelle. 
Table 1. Fits of the scaled forms of the various PEFs along the Relaxed Approach Paths (RAPs) for the $\mathrm{X}^{-}\left(\mathrm{H}_{2} \mathrm{O}\right), \mathrm{X}=\mathrm{F}, \mathrm{Cl}, \mathrm{Br}$, I, series ( $\xi=75$ and $\left.\eta=0.85\right) \cdot \chi^{2}$ denotes the weighted sum of squares of the fit, eq. (10). The number of ab initio points used in each fit is listed in parentheses.

\begin{tabular}{|c|c|c|c|c|c|}
\hline PEF & Parameters & $\begin{array}{l}\mathbf{F}^{-}\left(\mathbf{H}_{2} \mathbf{O}\right) \\
(28 \text { pts. })\end{array}$ & $\begin{array}{c}\mathbf{C l}^{-}\left(\mathbf{H}_{2} \mathbf{O}\right) \\
(27 \text { pts. })\end{array}$ & $\begin{array}{c}\mathbf{B r}^{-}\left(\mathbf{H}_{2} \mathbf{O}\right) \\
(29 \text { pts. })\end{array}$ & $\begin{array}{l}\mathbf{I}^{-}\left(\mathbf{H}_{2} \mathbf{O}\right) \\
\text { (30 pts. })\end{array}$ \\
\hline \multirow{2}{*}{$\begin{array}{c}\varepsilon(\mathrm{kcal} / \mathrm{mol}) \\
r_{m}(\AA)\end{array}$} & & 26.99 & 15.38 & 13.38 & 11.38 \\
\hline & & 2.451 & 3.103 & 3.298 & 3.547 \\
\hline \multirow[t]{2}{*}{$(e M)^{*}$} & $\alpha_{M}{ }^{*}$ & 5.3948 & 5.2428 & 4.804 & 4.7546 \\
\hline & $\begin{array}{l}\gamma_{2} \\
\chi^{2}\end{array}$ & $\begin{array}{c}2.0793 \\
2.39 \mathrm{E}-04\end{array}$ & $\begin{array}{c}2.0508 \\
2.02 \mathrm{E}-04\end{array}$ & $\begin{array}{c}2.1833 \\
8.03 \mathrm{E}-05\end{array}$ & $\begin{array}{c}2.1737 \\
1.10 \mathrm{E}-04\end{array}$ \\
\hline \multirow[t]{2}{*}{$(B e-6)^{*}-1$} & $\alpha$ & 0.7093 & 0.713 & 0.7269 & 0.726 \\
\hline & $\chi^{2}$ & $1.04 \mathrm{E}-02$ & $1.28 \mathrm{E}-02$ & $8.45 \mathrm{E}-03$ & 7.33E-03 \\
\hline \multirow[t]{3}{*}{$(g B e-\sigma)^{*}-1$} & $\alpha$ & 0.5423 & 0.5603 & 0.6124 & 0.6182 \\
\hline & $\delta$ & 2.1175 & 2.0912 & 2.2311 & 2.2197 \\
\hline & $\chi^{2}$ & $1.98 \mathrm{E}-04$ & $1.96 \mathrm{E}-04$ & $9.85 \mathrm{E}-05$ & $1.27 \mathrm{E}-04$ \\
\hline \multirow[t]{2}{*}{$(B e-6)^{*}-2$} & $\alpha$ & 2.2488 & 2.3339 & 2.2866 & 2.2731 \\
\hline & $\chi^{2}$ & $4.56 \mathrm{E}-03$ & 5.40E-03 & 3.09E-03 & 2.79E-03 \\
\hline \multirow[t]{3}{*}{$(g B e-\sigma)^{*}-2$} & $\alpha$ & 1.216 & 1.2298 & 1.3261 & 1.3427 \\
\hline & $\delta$ & 1.9929 & 1.9529 & 2.1091 & 2.1077 \\
\hline & $\chi^{2}$ & $4.00 \mathrm{E}-04$ & $2.78 \mathrm{E}-04$ & $8.02 \mathrm{E}-05$ & 1.17E-04 \\
\hline \multirow[t]{2}{*}{$(B-2)^{*}$} & $\gamma$ & $<0$ & $<0$ & $<0$ & $<0$ \\
\hline & $\chi^{2}$ & 7.30E-03 & $8.71 \mathrm{E}-03$ & 7.43E-03 & 7.51E-03 \\
\hline \multirow[t]{3}{*}{$(g B-2)^{*}$} & $\gamma$ & 9.802 & 9.3594 & 8.3145 & 8.1564 \\
\hline & $\delta$ & 1.8896 & 1.8052 & 1.9196 & 1.9162 \\
\hline & $\chi^{2}$ & $5.99 \mathrm{E}-04$ & $4.20 \mathrm{E}-04$ & $5.95 \mathrm{E}-05$ & $6.12 \mathrm{E}-05$ \\
\hline \multirow[t]{3}{*}{$(g B-2)^{*} \mid \delta=2$} & $\gamma$ & 9.6795 & 9.152 & 8.2247 & 8.0665 \\
\hline & $\delta$ & 2.0 & 2.0 & 2.0 & 2.0 \\
\hline & $\chi^{2}$ & $6.56 \mathrm{E}-04$ & $6.06 \mathrm{E}-04$ & 8.42E-05 & 8.49E-05 \\
\hline \multirow[t]{3}{*}{$(e B-2)^{*}$} & $\gamma$ & 10.2598 & 9.8345 & 8.708 & 8.5847 \\
\hline & $\zeta$ & 2.1036 & 2.0788 & 2.2011 & 2.1903 \\
\hline & $\chi^{2}$ & $2.14 \mathrm{E}-04$ & $1.83 \mathrm{E}-04$ & $5.29 \mathrm{E}-05$ & $7.45 \mathrm{E}-05$ \\
\hline \multirow[t]{3}{*}{$(e B-2)^{*} \mid \zeta=2$} & $\gamma$ & 10.584 & 10.0714 & 9.2804 & 9.1136 \\
\hline & $\zeta_{2}$ & 2.0 & 2.0 & 2.0 & 2.0 \\
\hline & $\chi^{2}$ & 2.91E-04 & 2.29E-04 & $3.23 \mathrm{E}-04$ & $2.85 \mathrm{E}-04$ \\
\hline
\end{tabular}


Table 2. Fits of the scaled forms of the various PEFs along the Relaxed Approach Paths (RAPs) for the $\mathrm{M}^{+}\left(\mathrm{H}_{2} \mathrm{O}\right), \mathrm{M}=\mathrm{Li}, \mathrm{Na}, \mathrm{K}, \mathrm{Rb}, \mathrm{Cs}$, series ( $\xi=75$ and $\eta=0.85$ ). $\chi^{2}$ denotes the weighted sum of squares of the fit, eq. (10). The number of $a b$ initio points used in each fit is listed in parentheses.

\begin{tabular}{|c|c|c|c|c|c|c|}
\hline PEF & Parameters & $\begin{array}{c}\mathbf{L i}^{+}\left(\mathbf{H}_{2} \mathbf{O}\right) \\
(54 \text { pts. })\end{array}$ & $\begin{array}{c}\mathbf{N a}^{+}\left(\mathbf{H}_{2} \mathbf{O}\right) \\
(52 \text { pss. })\end{array}$ & $\begin{array}{c}\mathbf{K}^{+}\left(\mathbf{H}_{\mathbf{2}} \mathbf{O}\right) \\
(59 \text { pts. })\end{array}$ & $\begin{array}{c}\mathbf{R b}^{+}\left(\mathbf{H}_{2} \mathbf{O}\right) \\
(63 \text { pts. })\end{array}$ & $\begin{array}{c}\mathbf{C s}^{+}\left(\mathbf{H}_{2} \mathbf{O}\right) \\
(68 \text { pts. })\end{array}$ \\
\hline$\varepsilon(\mathrm{kcal} / \mathrm{mol})$ & & 32.34 & 22.01 & 14.84 & 13.21 & 11.66 \\
\hline$r_{m}(\AA)$ & & 1.867 & 2.266 & 2.743 & 2.895 & 3.080 \\
\hline \multirow[t]{2}{*}{$(e M)^{*}$} & $\alpha_{M}{ }^{*}$ & 3.2005 & 3.7923 & 4.2426 & 4.3003 & 4.5025 \\
\hline & $\begin{array}{l}\gamma_{2} \\
\chi^{2}\end{array}$ & $\begin{array}{c}1.8575 \\
7.38 \mathrm{E}-06 \\
\end{array}$ & $\begin{array}{c}1.8475 \\
1.29 \mathrm{E}-05 \\
\end{array}$ & $\begin{array}{c}1.8188 \\
2.21 \mathrm{E}-05 \\
\end{array}$ & $\begin{array}{c}1.802 \\
2.66 \mathrm{E}-05 \\
\end{array}$ & $\begin{array}{c}1.7901 \\
3.45 \mathrm{E}-05 \\
\end{array}$ \\
\hline \multirow[t]{2}{*}{$(B e-6)^{*}-1$} & $\alpha$ & 0.8166 & 0.7916 & 0.7799 & 0.7839 & 0.7807 \\
\hline & $\chi^{2}$ & $1.61 \mathrm{E}-02$ & $1.30 \mathrm{E}-02$ & $1.47 \mathrm{E}-02$ & $1.53 \mathrm{E}-02$ & $1.69 \mathrm{E}-02$ \\
\hline \multirow[t]{3}{*}{$(g B e-\sigma)^{*}-1$} & $\alpha$ & 0.7779 & 0.7228 & 0.6739 & 0.6671 & 0.6436 \\
\hline & $\delta$ & 1.7946 & 1.8366 & 1.8294 & 1.8141 & 1.8088 \\
\hline & $\chi^{2}$ & 7.56E-05 & $2.46 \mathrm{E}-05$ & $1.92 \mathrm{E}-05$ & $2.20 \mathrm{E}-05$ & $2.47 \mathrm{E}-05$ \\
\hline \multirow[t]{2}{*}{$(B e-6)^{*}-2$} & $\alpha$ & 3.9515 & 3.5813 & 3.3906 & 3.3124 & 3.2807 \\
\hline & $\chi^{2}$ & 8.91E-04 & $1.35 \mathrm{E}-03$ & $1.77 \mathrm{E}-03$ & $1.72 \mathrm{E}-03$ & $1.96 \mathrm{E}-03$ \\
\hline \multirow[t]{3}{*}{$(g B e-6)^{*}-2$} & $\alpha$ & 2.6436 & 1.9579 & 1.6091 & 1.5778 & 1.4748 \\
\hline & $\delta$ & 1.9497 & 1.9012 & 1.8409 & 1.8249 & 1.7957 \\
\hline & $\chi^{2}$ & 4.78E-05 & $1.96 \mathrm{E}-05$ & $1.51 \mathrm{E}-05$ & $1.75 \mathrm{E}-05$ & $2.75 \mathrm{E}-05$ \\
\hline \multirow[t]{2}{*}{$(B-2)^{*}$} & $\gamma$ & $<0$ & $<0$ & $<0$ & $<0$ & $<0$ \\
\hline & $\chi^{2}$ & $5.24 \mathrm{E}-03$ & $3.12 \mathrm{E}-03$ & $3.24 \mathrm{E}-03$ & $3.08 \mathrm{E}-03$ & $3.37 \mathrm{E}-03$ \\
\hline \multirow[t]{3}{*}{$(g B-2)^{*}$} & $\gamma$ & 0.4129 & 3.1097 & 5.1903 & 5.2628 & 6.0687 \\
\hline & $\delta$ & 1.748 & 1.6218 & 1.533 & 1.49 & 1.4572 \\
\hline & $\chi^{2}$ & $1.87 \mathrm{E}-04$ & 4.73E-04 & $6.70 \mathrm{E}-04$ & 7.62E-04 & $8.38 \mathrm{E}-04$ \\
\hline \multirow[t]{3}{*}{$(g B-2)^{*} \mid \delta=2$} & $\gamma$ & $<0$ & 1.5836 & 3.6693 & 3.3189 & 4.2455 \\
\hline & $\delta$ & 2.0 & 2.0 & 2.0 & 2.0 & 2.0 \\
\hline & $\chi^{2}$ & $2.48 \mathrm{E}-04$ & 7.34E-04 & $1.42 \mathrm{E}-03$ & $1.51 \mathrm{E}-03$ & $1.94 \mathrm{E}-03$ \\
\hline \multirow[t]{3}{*}{$(e B-2)^{*}$} & $\gamma$ & 4.0267 & 6.093 & 7.3618 & 7.5324 & 8.0589 \\
\hline & $\zeta$ & 1.7753 & 1.8002 & 1.7972 & 1.7793 & 1.7795 \\
\hline & $\chi^{2}$ & $1.29 \mathrm{E}-04$ & $8.02 \mathrm{E}-05$ & 5.94E-05 & $6.24 \mathrm{E}-05$ & $6.09 \mathrm{E}-05$ \\
\hline \multirow[t]{3}{*}{$(e B-2)^{*} \mid \zeta=2$} & $\gamma$ & 2.3378 & 5.1726 & 6.5335 & 6.5738 & 7.1246 \\
\hline & $\zeta$ & 2.0 & 2.0 & 2.0 & 2.0 & 2.0 \\
\hline & $\chi^{2}$ & 3.63E-04 & $3.73 \mathrm{E}-04$ & 4.30E-04 & $5.08 \mathrm{E}-04$ & $5.63 \mathrm{E}-04$ \\
\hline
\end{tabular}




\section{Figure Captions}

Figure 1. The behavior of the $(B e-\sigma),(g B e-\sigma)$ relative to the (eM) PEF (left panels) and that of the $(B-2),(g B-2),(e B-2)$ relative to the $(e M)$ PEF (right panels). For each case the top(bottom) panels show the variation in the minimum and long(short)-range part when the short(minimum and long)-range parts are adjusted to be roughly the same to the degree that this is possible. The comparison with the $(e M) \mathrm{PEF}$ is chosen because of its superior performance for this class of interactions.

Figure 2. Fits of the $\mathrm{X}^{-}\left(\mathrm{H}_{2} \mathrm{O}\right), \mathrm{X}=\mathrm{F}, \mathrm{Cl}, \mathrm{Br}, \mathrm{I}$ ab initio PESs (filled circles) with the $(\mathrm{Be}$ 6), $(g B e-6),(B-2),(g B-2)$ and $(e B-2)$ PEFs. The fits were performed with $\xi=75$ and $\eta=0.85$ for all cases.

Figure 3. Fits of the $\mathrm{M}^{+}\left(\mathrm{H}_{2} \mathrm{O}\right), \mathrm{M}=\mathrm{Li}, \mathrm{Na}, \mathrm{K}, \mathrm{Rb}, \mathrm{Cs}$ ab initio PESs (filled circles) with the $(B e-6),(g B e-6),(B-2),(g B-2)$ and $(e B-2)$ PEFs. The fits were performed with $\xi=75$ and $\eta=0.85$ for all cases.

Figure 4. Effect of the choices of the ( $\xi$ and $\eta$ ) parameters on the value of $\chi^{2}$ shown for the $\mathrm{Br}^{-}\left(\mathrm{H}_{2} \mathrm{O}\right)$ case. Left panel: $\xi=0$ (for that value of $\xi$ the value of $\eta$ is irrelevant); emphasis on fitting the repulsive wall. Middle panel: $\xi=75$, $\eta=0.85$; emphasis on fitting the area around minimum and the long-range attractive part (currently used in the fits in Tables 1 and 2). Right panel: $\xi=1000, \eta=0.5$; emphasis on fitting the long-range attractive part.

Figure 5. Variation of the $(g B-2)^{*}$ PEF for different values of the parameters $\gamma$ and $\delta$, demonstrating that the first one mainly controls the short-range, repulsive wall, while the second the long-range parts of the potential. 

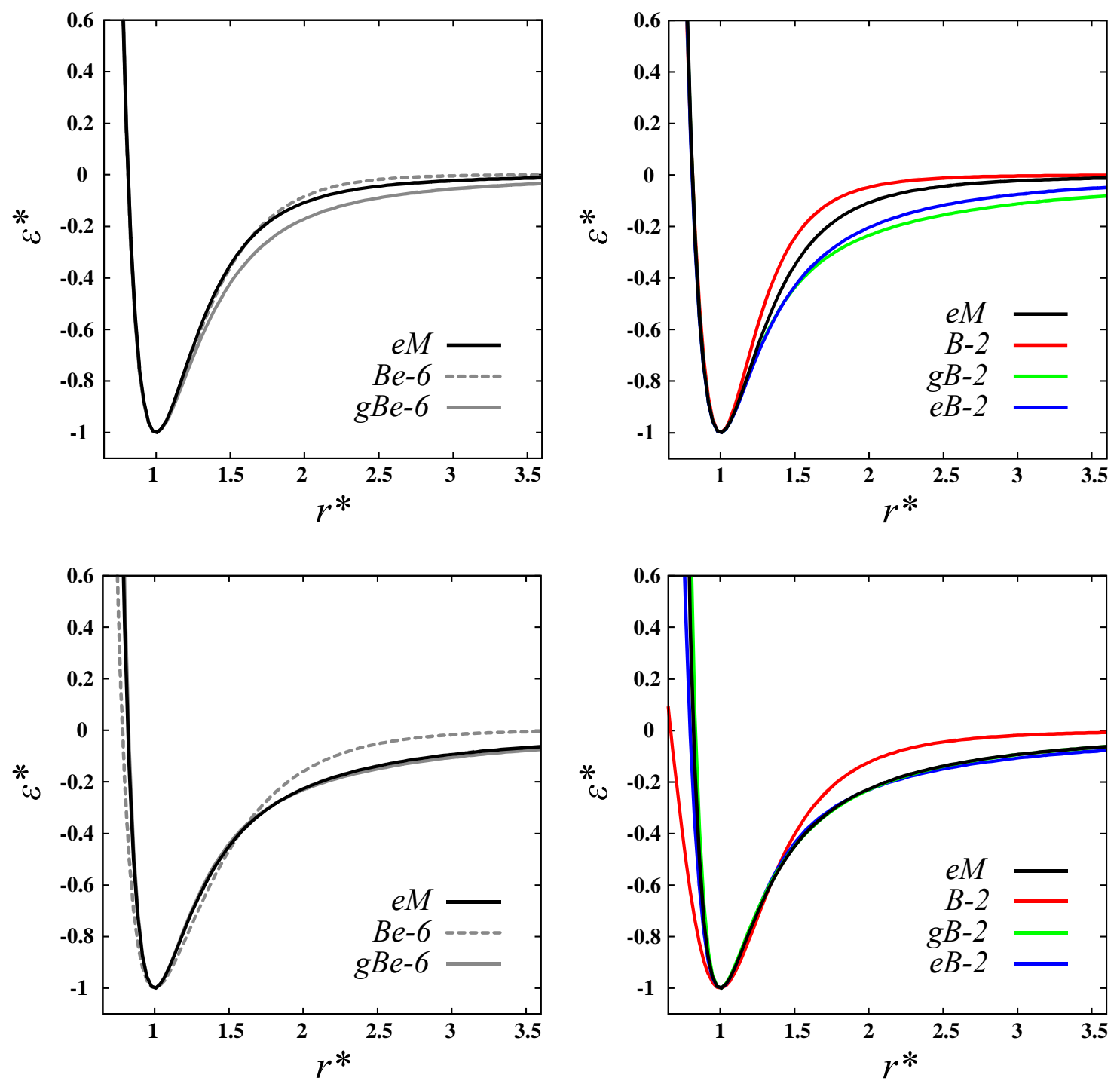

Figure 1 


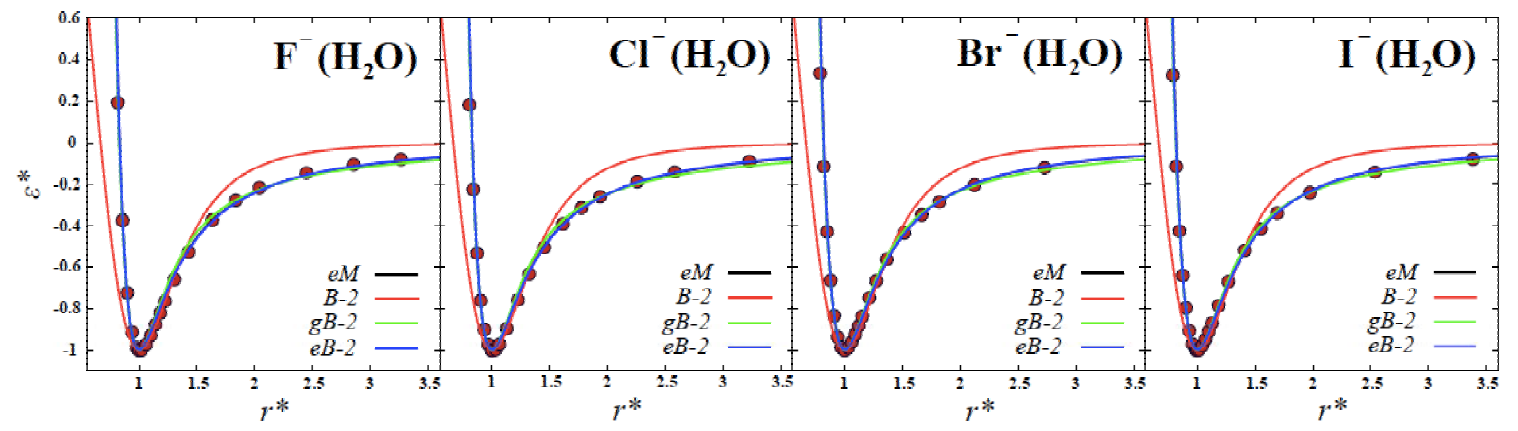

Figure 2 


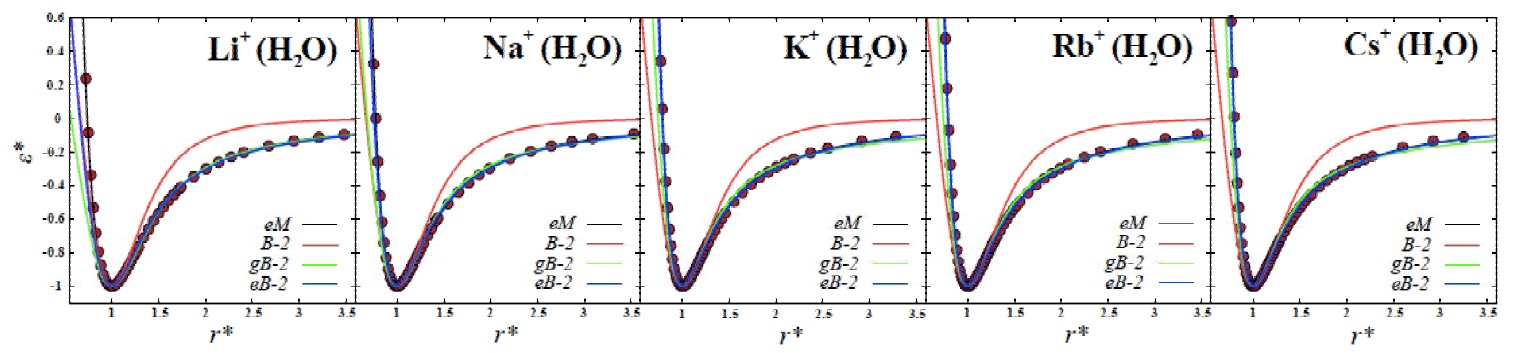

Figure 3 


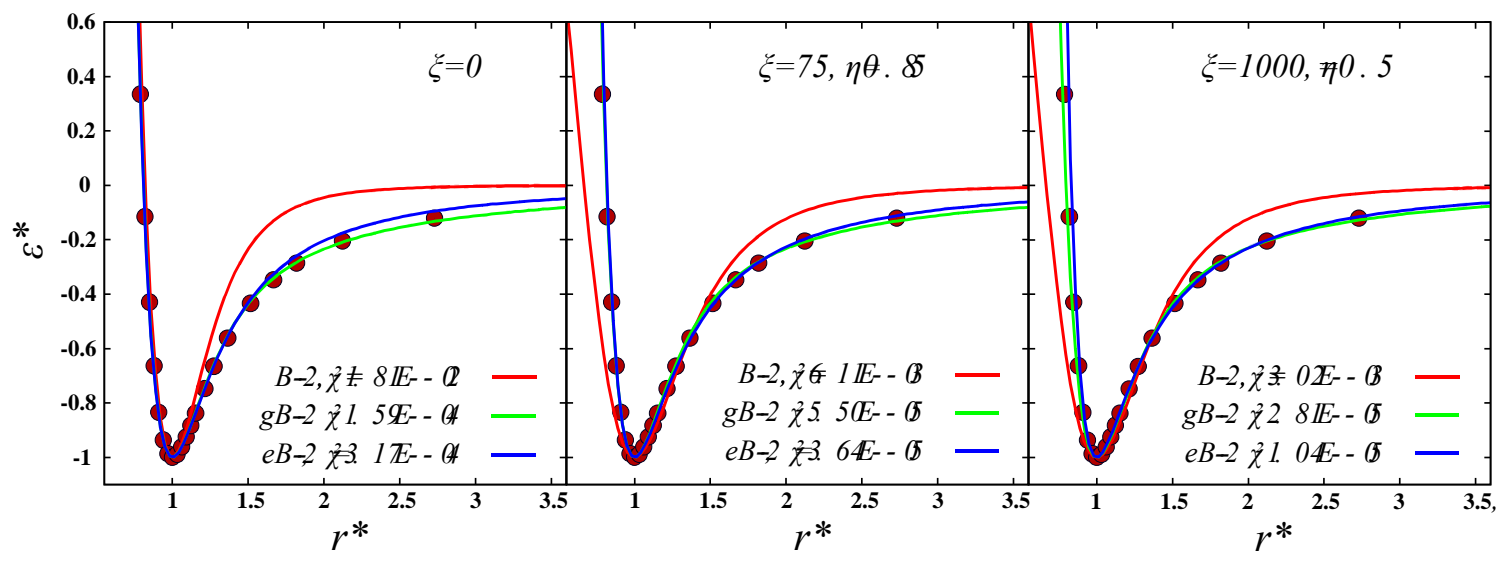

Figure 4 

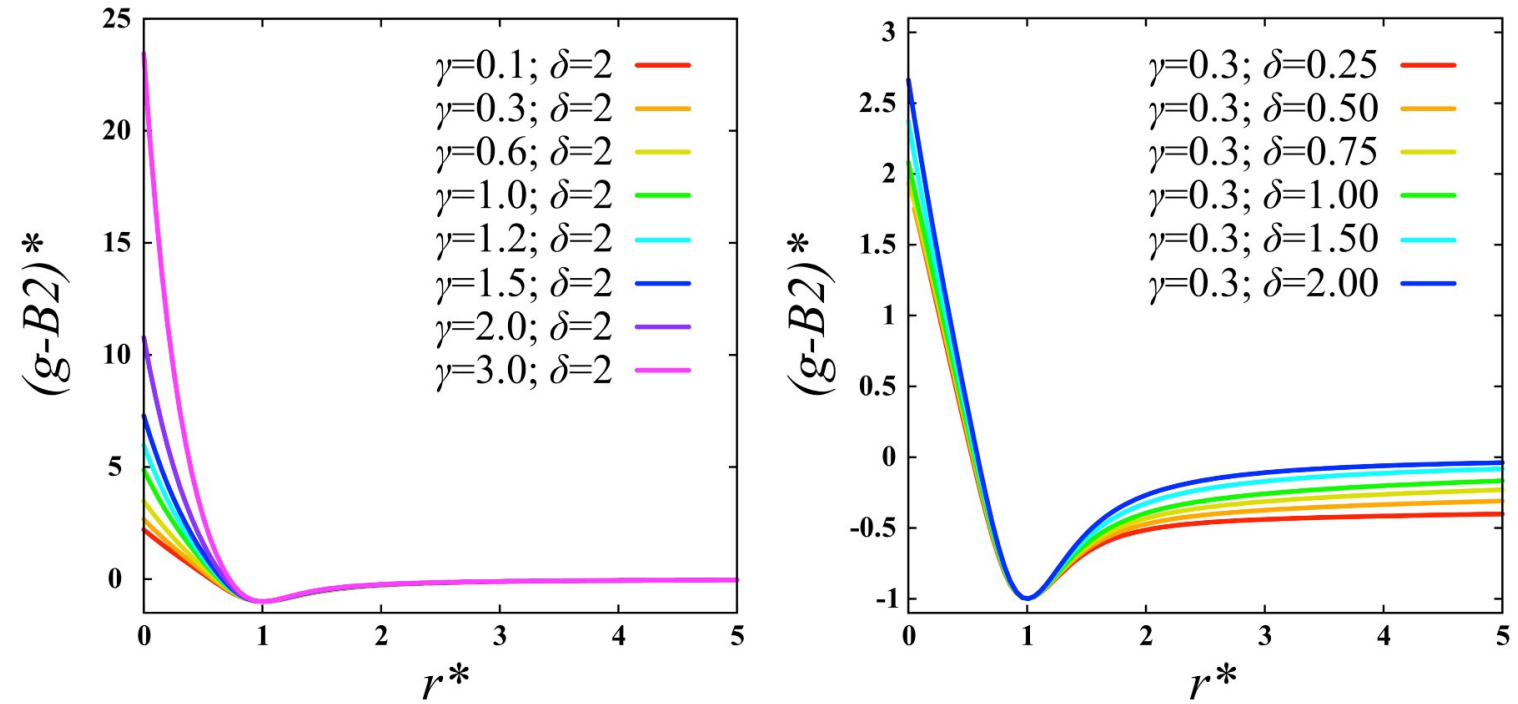

Figure 5 


\section{REFERENCES}

[1] R. A. Buckingham, Proceedings of the Royal Society of London. Series A, Mathematical and Physical Sciences 168 (1938) 264.

[2] J. E. Jones, Proceedings of the Royal Society of London. Series A 106 (1924) 463.

[3] J. E. Jones, A. E. Ingham, Proceedings of the Royal Society of London. Series A 107 (1925) 636.

[4] J. E. Lennard-Jones, Proceedings of the Physical Society 43 (1931) 461.

[5] M. Born, J. E. Mayer, Z. Physik 75 (1932) 1.

[6] M. Born Atomic Physics; Hafner Publishing Co.: New York, 1946.

[7] J. R. Errington, G. C. Boulougouris, I. G. Economou, A. Z.

Panagiotopoulos, D. N. Theodorou, J. Phys. Chem. B 102 (1998) 8865.

[8] J. R. Errington, A. Z. Panagiotopoulos, The Journal of Chemical Physics 109 (1998) 1093.

[9] J. R. Errington, A. Z. Panagiotopoulos, J. Chem. Phys. 109 (1998) 1093.

[10] J. R. Errington, A. Z. Panagiotopoulos, J. Phys. Chem. B 103 (1999) 6314.

[11] J. R. Errington, A. Z. Panagiotopoulos, J. Chem. Phys. 111 (1999) 9731.

[12] J. R. Errington, A. Z. Panagiotopoulos, J. Chem. Phys. 118 (2003) 7556.

[13] S. S. Xantheas, J. C. Werhahn, J. Chem. Phys. 141 (2014) 064117.

[14] L.-P. Wang, J. Chen, T. van Voorhis, Journal of Chemical Theory and Computation 9 (2013) 452.

[15] J. C. Werhahn, D. Akase, S. S. Xantheas, J. Chem. Phys. 141 (2014) 064118. 

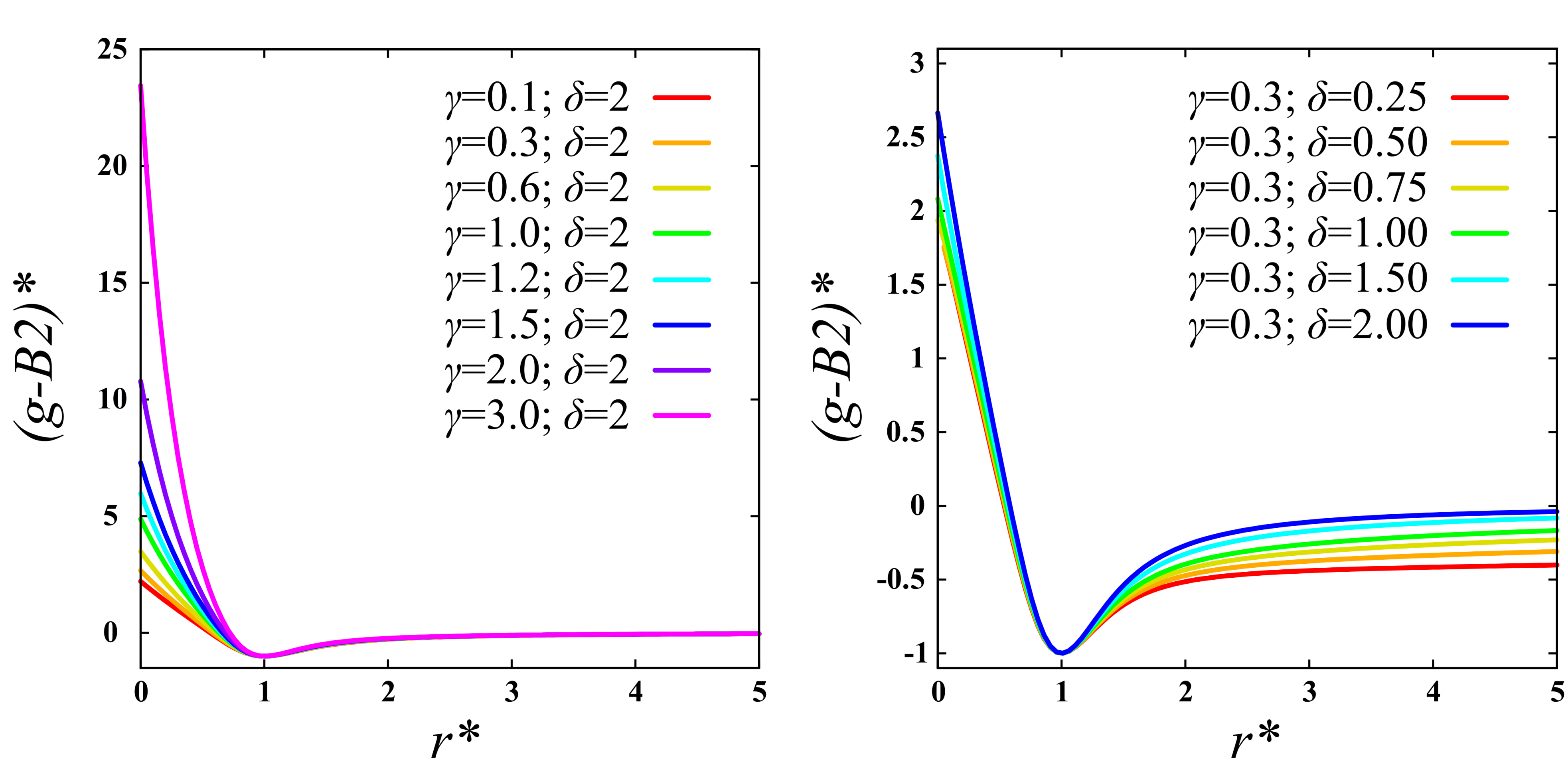